DOI: 10.17707/AgricultForest.65.2.05

\author{
Benji Brayan SILVA, \\ Charina Gracia BANAAY, Kevin SALAMANEZ ${ }^{1}$
}

\title{
TRICHODERMA-INDUCED SYSTEMIC RESISTANCE AGAINST THE SCALE INSECT (UNASPIS MABILIS LIT \& BARBECHO) IN LANZONES (LANSIUM DOMESTICUM CORR.)
}

\begin{abstract}
SUMMARY
Unaspis mabilis infestations have been causing huge economic losses to the lanzones industry in the Philippines since 2009. This study, therefore, seeks to determine the use of Trichoderma as myco-biocontrol agent to increase lanzones (Lansium domesticum) defense and resistance against this insect. Two experiments were designed to test for the protective and rescuing capacity of Trichoderma inoculation against the insect pest. For both experiments, leaf area, leaf count, scale insect populations and densities were observed over time. Additionally, to assess the phytochemical response of the plants, the concentrations of jasmonic acid, salicylic acid, total phenol content, and total flavonoid content were also measured in the rescuing capacity experiment. Results indicated that the plants inoculated with Trichoderma were performing better, showing lower populations and slower growth rate of the insect, than the uninoculated plants for both the protective and rescuing capacity experiments. Phytochemical analysis revealed the presence of tannins, phlobatannins, flavonoids, steroids, glycosides, and alkaloids in the leaves of L. domesticum. Total phenols and flavonoids showed decreasing concentrations as the infestation progressed, while the concentrations of jasmonic acid and salicylic acid in scaleinsect-infested plants were found to be influenced by the inoculation of the plants with Trichoderma.
\end{abstract}

Keywords: biocontrol, phytochemicals, Trichoderma, scale insect, systemic resistance

\section{INTRODUCTION}

A new species of armored scale insect, Unaspis mabilis Lit and Barbecho, was identified in the Philippines in 2014. Reports of outbreak-level infestations were first recorded in North Cotabato in 2009, and since then in different parts of the Luzon and Visayas regions (Lit and Barbecho, 2014). However, other reports dated the infestations as early as 2004 (Provido, 2007) although the pest was

\footnotetext{
${ }^{1}$ Benji Brayan Silva, Institute of Biological Sciences, College of Arts and Sciences, University of the Philippines Los Baños, 4031, PHILIPPINES; Charina Gracia Banaay (corresponding author: cbbanaay@up.edu.ph), Institute of Biological Sciences, College of Arts and Sciences, University of the Philippines Los Baños, 4031, PHILIPPINES; Kevin Salamanez, Institute of Chemistry, College of Arts and Sciences, University of the Philippines Los Baños, 4031, PHILIPPINES..

Notes: The authors declare that they have no conflicts of interest. Authorship Form signed online.
} 
misidentified as Lepidosaphes ulmi (Watson, 2015). The infestations were reported to have caused millions of pesos worth of losses in agricultural produce and hundreds of thousands of trees were already invaded in a municipality in North Cotabato alone. Methods of controlling the infestations, including spraying of commercially available chemical insecticides, have been proven ineffective. The research community in the Philippines has been seeking the use of alternative and biocontrol methods to solve or alleviate this problem, such as the use of scale insect-eating beetles Chilocorus nigrita and Chilocorus circumdatus (Brion, 2016; Watson, 2015; GMA News Online, 2008). This study explores the possible use of a fungal agent to induce plant resistance against the $U$. mabilis infestation.

Trichoderma spp. used as biocontrol agents against plant-infecting pathogens have been recorded since the 1920s. Earlier studies of the use of myco-biocontrol agents, such as Trichoderma, focused on increasing their efficiency to directly attack a fungal or bacterial plant pathogen, or even living insects, through competition for resources and space, parasitism and antibiotics production (Harman et al., 2008; Harman, 2006). Recent studies, however, argued that the direct effects of the use of Trichoderma as a biocontrol agent is as important, if not less, as its indirect effect to the overall plant defenses (Harman et al., 2004). This is due to the recent discoveries on the capacity of Trichoderma to elicit systemic and/or localized acquired or induced resistance, and therefore inducing the primed state of plants (Saldajeno et al., 2014). During the plant primed state, its defense system is immediately and/or more aggressively activated following exposure to either/both biotic and/or abiotic stress (Frost et al., 2008; Conrath et al., 2006).

This study aims to determine whether Trichoderma inoculation of seedlings can help protect against and/or alleviate scale insect infestation of lanzones. It is also of interest in this study to observe the relationships among the overall plant growth, jasmonic acid (JA), salicylic acid (SA), and some secondary metabolites concentrations, and the insect population in order to hypothesize on probable defense mechanisms.

\section{MATERIALS AND METHODS \\ The Experimental Set-up}

In order to evaluate the feasibility of using Trichoderma spp. as a mycobiocontrol agent against the lanzones scale-insect, Unaspis mabilis, two set-ups were utilized in this study. The first set-up was used to determine the protective effect of inoculating uninfested lanzones seedlings with Trichoderma spp. against the eventual attack of the scale insect. Meanwhile, the second set-up was used to directly establish the use of Trichoderma to rescue lanzones plants that are already infested with $U$. mabilis.

Protective Capacity: Eighteen (18) healthy uninfested one-and-a-halfyear-old lanzones seedlings were randomly grouped into two treatments. The 
first treatment was inoculated with Trichoderma microbial inoculant according to manufacturer's instructions, while the second treatment was not.

A month after the inoculation, both treatments were exposed to infestation of Unaspis mabilis by placing the seedlings inside an insect net together with plants that are already infested by the same insect. The seedlings were arranged inside the net in random order to minimize the effect of wind direction, and other dispersal parameters to the favorability of transfer of scale insect to the test plants.

After the one-month exposure of the healthy seedlings to the insect, the two groups were isolated by placing each group in separate nets to monitor the morphological changes in the plants, as well as the population changes of $U$. mabilis. The seedlings were inspected for the same parameters mentioned above every month for another three months.

All the leaves of the 18 seedlings belonging to the two treatments were photographed for morphological assessment. Leaf area of each of the leaves of the plants, count of adult female scale insect (FSIC) on each of the leaves, and the area covered by the male scale insect pupae (MSIC) were determined through assessment of photographs using ImageJTM. The leaf count of each of the seedlings was also noted. The density of the adult female scale insect (FSID) and percent cover of male scale insect pupae (MSID) were computed.

Rescuing Capacity: One hundred thirty-two (132) scale insect-infested one-and-a-half-year-old seedlings were randomly divided into six equal groups. Initial data on leaf count, leaf area, FSIC, FSID, MSIC, and MSID from 50 randomly selected leaves in each group were noted. In addition, phytochemical screening, and quantification of total phenols, total flavonoids, and phytohormones JA and SA were also assessed prior to treatment application.

After the initial data gathering, the six groups were randomly assigned under two treatments namely inoculated, and not inoculated with Trichoderma, in triplicates. Each treatment group was placed inside an insect net to avoid cross infestation of scale insect between replicates and treatments, and to avoid the introduction of outside pest population to the experimental set-up. The same set of infestation parameters, and morphological and phytochemical parameters were measured every two weeks for the next six weeks.

The Trichoderma microbial inoculant (BIOSPARK TM) used in this study was obtained from Dr. Virginia Cuevas of the Institute of Biological Sciences, University of the Philippines Los Baños. Five grams of the inoculant was used for each seedling following the established application protocol for BIOSPARK TM administration.

\section{Phytochemical Screening and Quantification}

To determine some of the groups and classes of phytochemicals that are present in lanzones leaves which may be involved in the plant's defense mechanisms, an initial phytochemical screening was conducted wherein the presence of tannins, phlobatannins, saponins, flavonoids, steroids, and glycosides 
were determined according to Evans (1989), while the presence of alkaloids and anthraquinones were tested according to Cock (2011).

Ten grams of leaves were randomly collected from each of the replicates of the two treatments. The leaves were cut into pieces approximately $2 \mathrm{~cm}^{2}$ each. The pieces of leaves were mixed, and $7.0 \mathrm{~g}$ was randomly selected and then, it was extracted with $35.0 \mathrm{~mL}$ of methanol using a blender. Another gram of leaves randomly selected from the same group was extracted with $20.0 \mathrm{~mL}$ of distilled water. These extraction procedures were done separately for each replicate of the treatments. The methanolic extract was centrifuged at $3000 \times g$ for five minutes, while the aqueous extract was centrifuged at $10,000 \times g$ for ten minutes.

Jasmonic acid Extraction and Quantification: To extract the plant hormone jasmonic acid (JA) endogenous to the leaves sampled a modified method by Dhandukia and Thakkar (2008) was used for the methanolic extract described above.

Briefly, the extracts were acidified to $\mathrm{pH} 3.0$ with $6 \mathrm{~N}$ hydrochloric acid $(\mathrm{HCl})$. The acidified solution was evaporated to dryness at $35^{\circ} \mathrm{C}$. The dried products were re-dissolved in $1.0 \mathrm{~mL}$ of methanol-JA solution with a concentration of $100 \mu \mathrm{g} / \mathrm{mL}$. This served as the internal standard of JA in the sample.

Aluminum-backed silica gel $60 \mathrm{~F}_{254}$ TLC foils $(14 \times 10 \mathrm{~cm})$ with $200 \mu \mathrm{m}$ layer thickness purchased from Sigma-Adrich Singapore were prepared also according to Dhandukia and Thakkar (2008). Thirteen lanes that are one cm apart were made in each TLC plate.

The re-dissolved dried extracts were loaded in the pre-conditioned TLC foils using a $10-\mu \mathrm{L}$ pipette. One microliter $(1.0 \mu \mathrm{L})$ of the sample was loaded in triplicate. Two samples were loaded in each plate.

Known concentrations of the standard solutions of JA were made by dissolving $100.0 \mathrm{mg}$ of jasmonic acid in methanol. Several lanes in each of the TLC plates were loaded with the JA solutions to achieve $0.1 \mu \mathrm{g} / \mathrm{band}$ (2 lanes), $0.2 \mu \mathrm{g} / \mathrm{band}$ (2 lanes), $0.4 \mu \mathrm{g} / \mathrm{band}$ (2 lanes), and $0.6 \mu \mathrm{g} / \mathrm{band}$ (1 lane). After loading the standards and samples onto the TLC plates, the plates were allowed to stand at $25^{\circ} \mathrm{C}$ for 5 to 10 minutes for drying before chromatogram development.

After drying, the TLC plates loaded with the appropriate samples and standards were placed inside a pre-saturated chamber with the mobile phase consisting of chloroform: ethyl acetate: acetone: acetic acid in 40:8:5:1 ratio (Ueda and Miyamoto, 1994). The average development time was 25 minutes.

After development, the plates were air-dried for 10 minutes. To visualize the chromatogram, $5.0 \mathrm{~g}$ of iodine crystals was placed in a cylindrical container with a height of $10.0 \mathrm{~cm}$ and a diameter of $10.5 \mathrm{~cm}$, and the dried plates were then exposed to iodine vapor for $12 \mathrm{~min}$.

After the exposure to the iodine vapor for visualization, the TLC plates were scanned to produce electronic image copies of the chromatograms. 
Quantitative analyses were performed on the images of the chromatograms using JustTLC v. 4.0.3, a TLC analysis software by Sweday.

The band volume and the known concentrations of the standard were plotted to construct a standard curve. The concentrations of the JA bands in the samples were computed based on the standard curve produced.

Salicylic Acid Quantification: Salicylic acid quantification was performed on the aqueous extract described above according to Warrier, Paul, and Vineetha (2013). The measurement of the concentration of flavonoids in the sample was expressed as $\mu \mathrm{g}$ SA per gram leaves ( $\mu$ g SA/g leaves). Standard curves were made using known concentrations of salicylic acid.

Total Phenolics and Flavonoids Quantifications: The methanolic extracts were also quantified for total phenolics and flavonoids according to the protocols of Singleton, Orthofer, and Lamuela-Raventos (1999) and Kiranmai, Kumar and Ibrahim (2011), respectively. Standard curves of gallic acid and quercetin were also prepared. The concentration of the total phenolics of the sample was expressed as gallic acid units per gram leaves (GA/g leaves), while the concentration of the flavonoids in the same sample was expressed as quercetin unit per gram leaves (QU/g leaves).

\section{Statistical Analysis}

For the comparison of the two treatments every observation period for significance of difference, Shapiro-Wilk Test for normality and Mann-Whitney U Test were used. For the correlation analyses, Doornik-Hansen Multivariate Test for normality was used. Pearson's correlation was used when the data satisfied the assumption of normality, otherwise Spearman's correlation was used.

\section{Trichoderma Protective Capacity}

\section{RESULTS AND DISCUSSION}

Assessments of the morphological characteristics of the seedlings belonging to the treatments with and without Trichoderma were done for the set up for the determination of the protective capacity of the Trichoderma spp.

Figure 1A shows that in the beginning of the experiment, there was no significant difference between the number of leaves of the seedlings in the two treatments. Meanwhile, seedlings inoculated with Trichoderma showed significantly higher leaf counts in all succeeding observation periods $(\mathrm{p}=0.040$, $\mathrm{p}=0.010$, and $\mathrm{p}<0.0005$ for Months 2, 3, and 4, respectively).

The ability of the seedlings to produce more leaves reflects the capacity of the plant to grow even in the presence of an insect pest. Increased growth, specifically in biomass yield, in plants inoculated with Trichoderma has been demonstrated in a number of literature including Zarate et al. (2015), and Harman (2000) among other studies. This is particularly highly correlated to the ability of these plants with Trichoderma as their mycosymbiont to have a more efficient nutrient uptake and utilization (Harman et al., 2008; Harman, 2001, 2000), as well as the upregulation of genes supporting more rapid growth (Harman and Shoresh, 2007). It has also been demonstrated that the leaves have an increased 
greenness that is hypothesized to be related to an increased photosynthetic rate thereby allowing the plants to create more resources for itself that is much needed for its own growth (Harman et al., 2008; Harman and Shoresh, 2007; Harman, 2000).
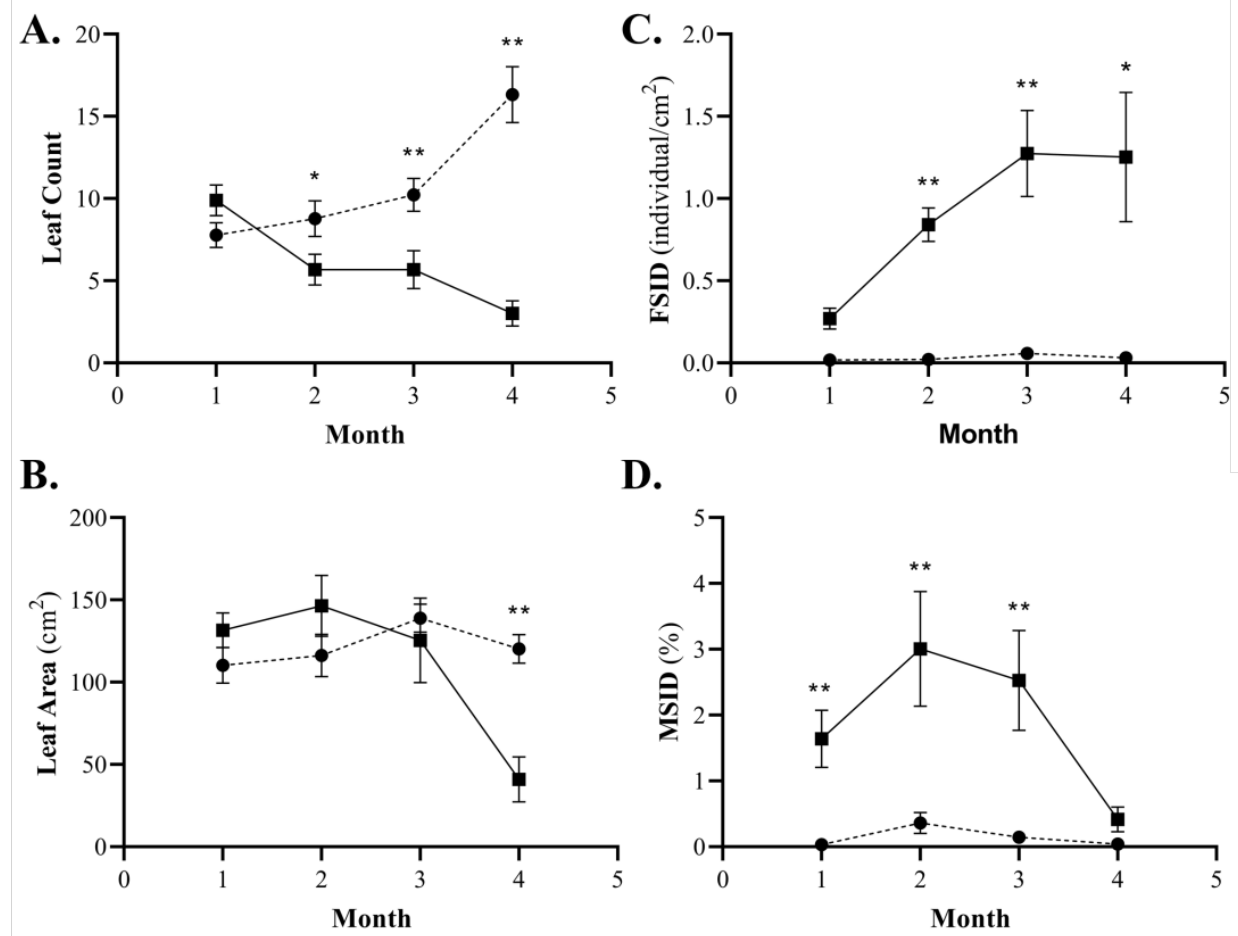

D.

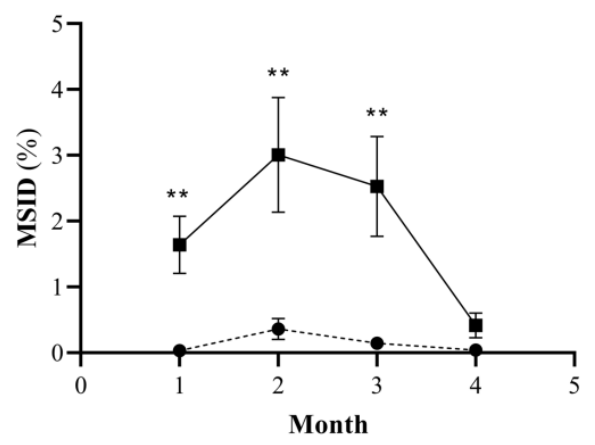

- with Trichoderma

- without Trichoderma

Figure 1. Comparisons between average leaf counts (A), leaf areas (B), female scaleinsect densities (C), and male scale-insect densities (D) of the two treatments (with Trichoderma \& without Trichoderma) over the four-month observation period [(Mean $\pm \mathrm{SEM})$; ${ }^{*}$ shows that $\mathbf{p} \leq \mathbf{0 . 0 5}$; ${ }^{* *}$ shows that $\left.\mathbf{p} \leq \mathbf{0 . 0 1}\right]$

On the other hand, it has been observed that there was no significant difference in the area of the leaves (Fig. 1B) of the seedlings from the two treatments up until the fourth month ( $p=0.004)$. However, on the sixth month, a highly significant difference was observed. This is due to the continuous loss of the older leaves of the seedlings in the treatment without Trichoderma. The much younger and smaller leaves are therefore the ones that were observed, thereby resulting in a significantly smaller leaf area.

Observation of the insect population showed that from the second up to the last observation period (Fig. 1C), FSIDs were observed to be significantly lower 
for the seedlings with Trichoderma $(\mathrm{p}<0.0005$ for Months 2 and 3, and $\mathrm{p}=$ 0.012 Month 4). It is also noteworthy to mention that the FSIDs of the seedlings with Trichoderma did not significantly increase over the four months of observation $(\mathrm{p}=1.000)$, while the seedlings without Trichoderma had a significant, generally increasing trend of FSIDs over the same period $(p=0.01)$.

Figure 1D shows that the treatment without Trichoderma has higher MSIDs from the first observation period up until the third month $(\mathrm{p}<0.005)$. During the last observation period (Month 4) no significant difference were observed which may be attributed to the effect of drastic decline of both leaf count (Fig. 1A) and leaf area (Fig. 1B) of the uninoculated plants.

It is clear from the results that Trichoderma inoculation was able to protect the lanzones seedlings from damaging levels of infestation in the leaves even if it was inoculated only in the roots. This is probably related to the capacity of the Trichoderma inoculant to potentiate the plant for a faster and stronger defense hence preventing the rapid increase in pest population. It has been shown that Trichoderma inoculation stimulates the jasmonic acid (JA)-dependent defense responses (Contreras-Cornejo et al., 2018; Martinez-Medina et al., 2013; Contreras-Cornejo et al., 2011), which is partly responsible for the initiation of the cascade of biochemical defense responses of plants against a wide array of biotic stresses. The inoculation of Lansium domesticum seedlings with Trichoderma before the insect attack possibly increased the sensitivity and responsiveness of the seedlings to jasmonic acid, as demonstrated by Pieterse et al. (2000).

\section{Trichoderma Rescuing Capacity}

Observations of the leaf counts of the seedlings belonging to the two treatments showed no statistical difference (Fig. 2A). However, leaf areas of the plants belonging to the treatment with Trichoderma are significantly broader during the fourth and sixth week of observation with $\mathrm{p}=0.001$ and $\mathrm{p}=0.034$, respectively (Fig. 2B). In addition, only the treatment with Trichoderma was also observed to exhibit a statistically significant increase in the average leaf area growth throughout the observation period $(\mathrm{p}=0.006)$.

In addition, Figures 2C and 2D show statistically significant reduction in FSID and MSID in infested plants inoculated with Trichoderma clearly showing the ability of the inoculant to alleviate the effects of the infestation. These observations alone demonstrate the advantage of Trichoderma inoculation to infested plants. In separate studies of Lo and Lin (2002), and Yedidia et al. (2001), leaf area of cucurbitaceous plants was shown to be larger in treatments inoculated with Trichoderma. This increase in leaf area is beneficial for the plants since it also increases the amount of intercepted light, thus increasing photosynthetic activity and, consequently, plant productivity. Increased productivity may provide the necessary "fuel" for increased production of metabolites for defense response and possibly compensate for losses incurred from insect feeding. 

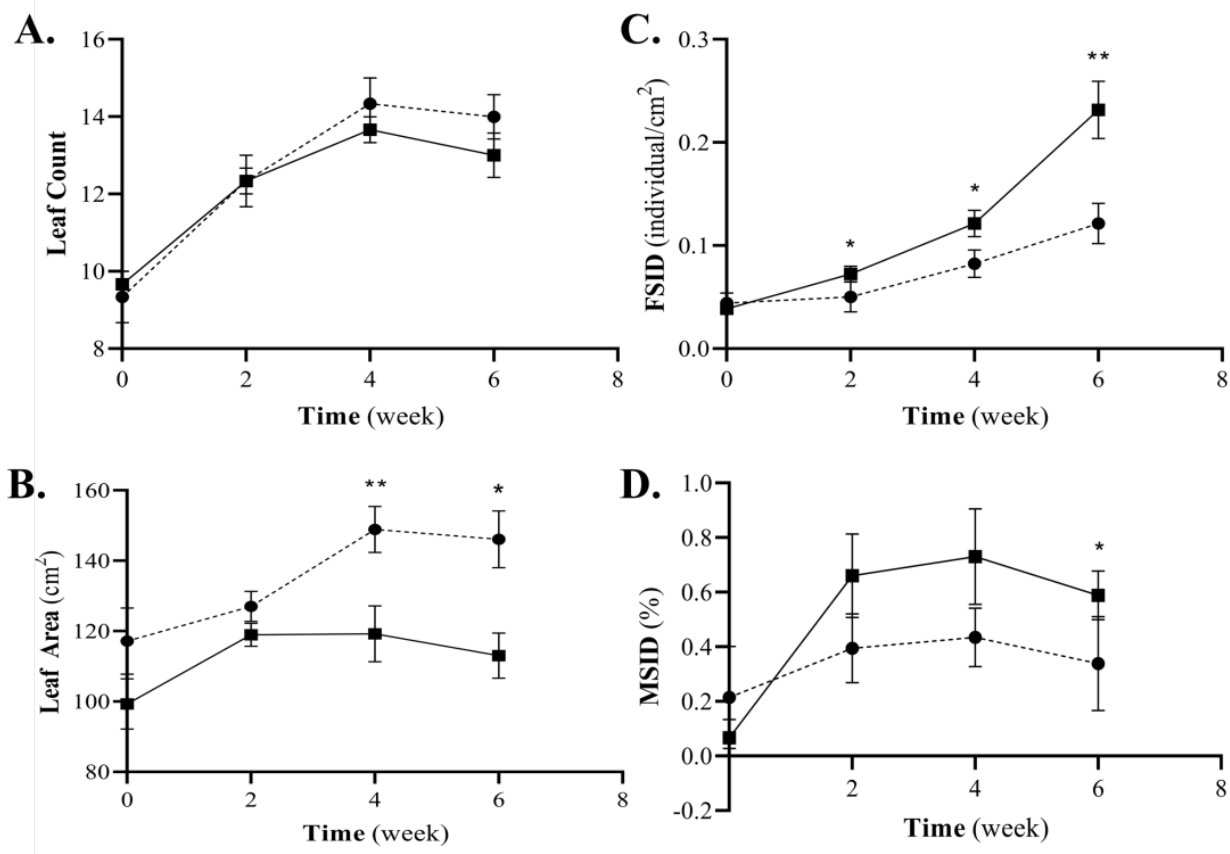

-. with Trichoderma

- without Trichoderma

Figure 2. Comparisons between average leaf counts (A), leaf areas (B), female scaleinsect densities (C), and male scale-insect densities (D) of the two treatments (with Trichoderma \& without Trichoderma) over the six-week observation period [(Mean \pm SEM); ${ }^{*}$ shows that $\mathbf{p} \leq \mathbf{0 . 0 5}$; ** shows that $\mathbf{p} \leq \mathbf{0 . 0 1}$ ]

To understand the mechanism behind these observed differences between the two treatments, phytochemical tests were also done. The phytohormones salicylic acid (SA) and jasmonic acid (JA) were quantified. Additionally, after assessing for the presence or absence of eight major groups of plant metabolites (Table 1), concentrations of flavonoids and phenols were quantified.

Table 1. Initial phytochemical screening of Lansium domesticum leaves

\begin{tabular}{|c|c|c|c|c|c|c|c|c|c|c|}
\hline \multicolumn{2}{|c|}{ Alkaloids } & \multicolumn{2}{|c|}{ Anthraquinones } & \multirow{2}{*}{ Flavonoids } & \multirow{2}{*}{ Glycosides } & \multirow{2}{*}{ Phlobatannins } & \multirow{2}{*}{ Saponins } & \multirow[b]{2}{*}{ Steroids } & \multirow[b]{2}{*}{ Tannins } & \multirow[b]{2}{*}{ Terpenoid } \\
\hline Mayer & Wagner & Ajayeoba & Kumar & & & & & & & \\
\hline$(+)$ & $(+)$ & $(-)$ & $(-)$ & $(+)$ & $(+)$ & $(+)$ & $(-)$ & $(+)$ & $(+)$ & $(+)$ \\
\hline
\end{tabular}

The qualitative screening showed presence of tannins, phlobatannis, flavonoids, steroids, glycosides, terpenoids, and alkaloids, which narrowed down the possible groups of Lansium domesticum secondary metabolites that can contribute to its general plant defense against the insect pest. Based on these results and the availability of methods and equipment that can be used for 
quantification, it was decided that the total phenolic content and flavonoid content of the leaves of the plants be quantified.

Total phenolic content was chosen because this class of compounds is one of the most diverse of all the classes of plant secondary metabolites. This includes the tannins, quinone, and flavonoids, among other groups that can function as herbivore deterrent, or may either kill or retard the development of an herbivore (War et al., 2012; Harborne, Baxter, and Moss 1999). In addition, the flavonoid group was also chosen to be quantified as a subset of the phenol class that has the capacity to protect plants against pests by influencing the insect's behavior, growth, and development. Some flavonoids were also observed to have cytotoxic properties and they can interact with other enzymes by complexation (War et al., 2012).

Quantification of SA (Fig. 3A) showed that SA increased over time in both treatments. The coefficients of linearity for the trends of SA concentration was $0.907(\mathrm{p}<0.0001)$ and $0.515(\mathrm{p}=0.086)$ for with and without Trichoderma, respectively. Studies on the differences of expressions of defense-related genes showed that biotrophic pathogens and phloem-feeding insects primarily activate the SA-dependent defense pathway, while the pathogens and herbivores causing minimal tissue damage activate the JA/ET pathway (Thaler, Agrawal and Halitschke, 2010; Smith, De Moraes and Mescher, 2008; Kempema et al., 2007; Thompson and Goggin, 2006; Glazebrook, 2005; Kaloshian and Walling, 2005; Rodriguez-Saona and Thaler, 2005; Rojo, Solano and Sanchez-Serrano 2003; Moran and Thompson, 2001).

In this study, the observed increase of SA in lanzones plants as infestation of $U$. mabilis progresses over time demonstrates similarity to other studies on phloem-feeding insects activating the SA-dependent defense pathway. According to the "decoy defense hypothesis" by Zhu-Salzman et al. (2004) on their study of the green bug aphid (Schizapis graminum) in sorghum (Sorghum bicolor), certain insect pests take advantage of the antagonistic interaction between JA and SA in order to increase their establishment on their hosts. The "decoy defense" was likewise observed in studies on Arabidopsis infested with Bemesia tabaci type B or silverleaf whitefly (Kempema et al., 2007; Zarate, Kempema and Walling, 2007). In these separate studies, silver leaf whitefly leveraged the antagonistic interaction of SA- and JA- signaling pathways as it causes strong induction of the SA pathway to suppress the JA pathway that can launch a more effective defense response against the infestation. Walling (2008) explained that phloem-feeding insects are able to deceive the host and suppress effective defense. By taking advantage of the negative crosstalk between the SA and JA pathways, the insects are able to inhibit the more effective JA-controlled defenses by stimulating the less effective SA-controlled defenses that inhibit the JA pathway.

Meanwhile, consistent with Zarate, Kempema and Walling (2007) and Kempema et al. (2007), the concentrations of JA (Fig. 3B) in the uninoculated seedlings showed no significant increase during the observation period 
$(\mathrm{p}=0.052)$. The association of JA concentration and time (e) in treatment without Trichoderma is $0.086(\mathrm{p}=0.79)$.

A.

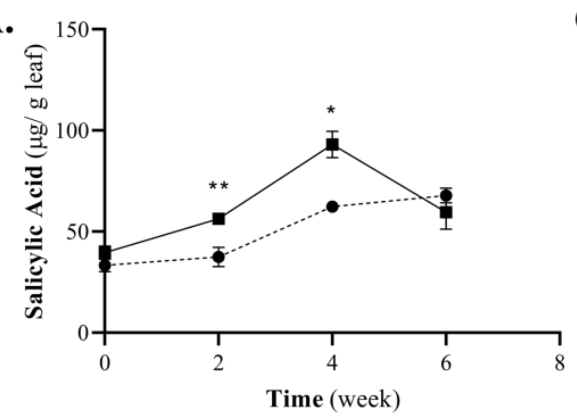

B.

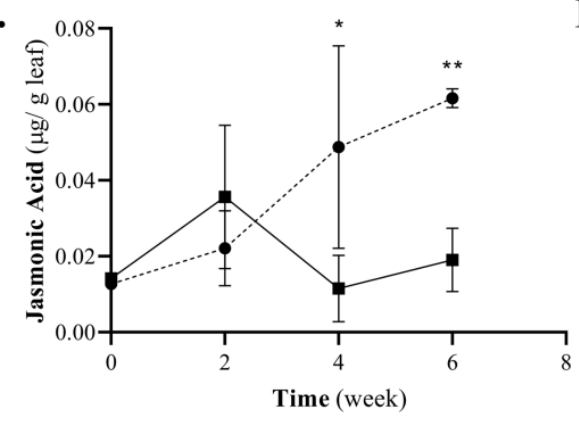

C.

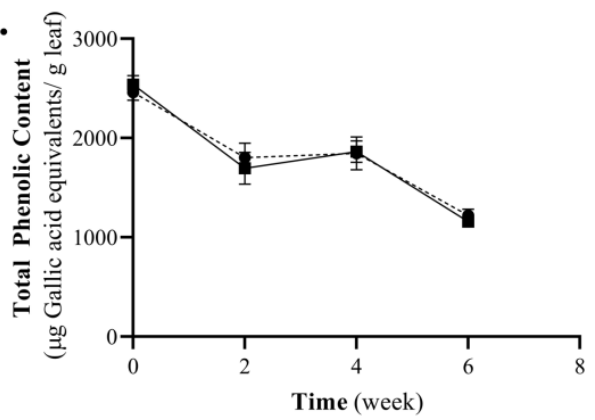

D.

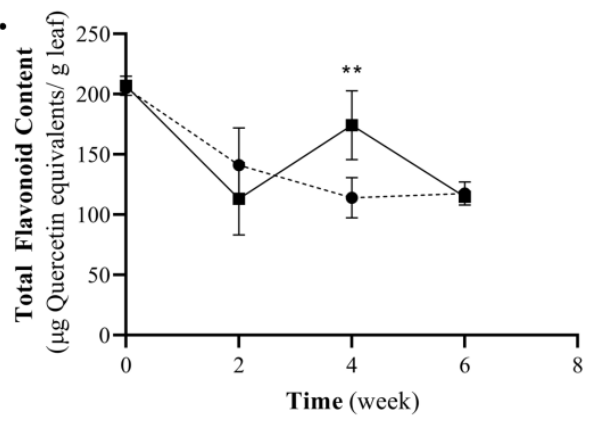

- with Trichoderma

- without Trichoderma

Figure 3. Comparisons between concentrations of jasmonic acid (A), salicylic acid (B), total phenolic content (C), and total flavonoid content (D) of the two treatments (with Trichoderma \& without Trichoderma) over the six-week observation period [(Mean \pm SEM); * shows that $\mathbf{p} \leq \mathbf{0 . 0 5}$; ** shows that $\mathbf{p} \leq \mathbf{0 . 0 1}$ ]

In addition, it was observed that uninoculated plants had significantly higher concentration of SA during the second and third observation periods ( $<0.0005$ for Week 2, and $p=0.040$ for Week 4). Earlier study by Zhou et al. (2018) showed similar results. Lower SA concentration was observed in Trichoderma gamsii inoculated Arabidopsis plants that are subsequently infested with Trichoplusia ni than those that were not inoculated. Contreras-Cornejo et al. (2018) also reported reduced methyl salicylate in plants inoculated with Trichoderma atroviride and challenged with Spodoptera frugiperda as compared to the plants that were also challenged with the same insect but are uninoculated by the T. atroviride.

On the other hand, concentrations of JA for the seedlings with Trichoderma during the six-week observation increased significantly $(\mathrm{p}=0.001)$ with $\mathrm{R}=0.687$ ( $\mathrm{p}=0.0136)$. Specifically, JA concentrations were found to be 
significantly higher in plants that were inoculated with Trichoderma compared to those that were uninoculated in Week $4(\mathrm{p}=0.012)$ and Week $6(\mathrm{p}<0.001)$. These observations suggest that in the absence of Trichoderma inoculation, SA accumulates while JA remains unchanged in infested lanzones plants. Meanwhile, relative to the treatment without inoculation, Trichodermainoculated plants accumulate SA slower and JA faster. Similarly, the observations of Contreras-Cornejo et al. (2018) indicate that, in their fungusplant-insect model, SA-mediated defense responses are attenuated while JA concentration is increased. Drawing from other studies, the same authors (Contreras-Cornejo et al., 2018) noted that their observations, similar to the observations in this study, [can] "not be a coincidence but a demonstration of a similar defense time-course patter[n] of plants challenged with different species of Trichoderma (p. 8)." In extension, these observations suggest that Trichoderma inoculation in plants provides a counter mechanism against the "decoy defense" of some phloem-feeding insects hiding behind a "hacked" SAmediated defense response.

Quantifications of the total phenolic content of the leaves of the seedlings belonging to both treatments, meanwhile, show a very strongly decreasing linear trend over time (Fig. 3C).

Time and total phenolic concentrations of the leaves in set-ups with and without Trichoderma have correlation coefficient $\mathrm{R}$ values of $-0.873(\mathrm{p}=0.0002)$ and $-0.862(\mathrm{p}<0.0001)$, respectively. No significant difference in phenolic concentration between the two treatments in any of the observation periods was observed. In addition to the reduced SA concentration, Zhou et al. (2018) also observed no significant difference in total phenolic between T. gamsii-inoculated and uninoculated T. ni-infested Arabidopsis plants.

However, the total flavonoid concentrations over the six-week time period varied between the two treatments (Fig. 3D). During the third observation period (Week 4), the seedlings inoculated with Trichoderma had a significantly lower concentration of flavonoid $(\mathrm{p}=0.005)$. However, for first, second, and last observation periods, no significant difference was observed. In general, the flavonoid concentration was decreasing over time.

With the observations of decreasing concentrations of phenols and flavonoids in both treatments, it can be hypothesized that other defense response metabolites that are outside the phenol group may have been used by the lanzones plants and that a different set of metabolites was induced instead. In a paper by Xiao et al. (2019), they observed that herbivory by a phloem-feeding insect does not show any induction of the total phenolics and total flavonoids in the host plant unlike chewing herbivores that cause significant increases in total phenolics and flavonoids compared with control plants.

Alternatively, Close and McArthur (2002) suggested that perhaps there is a need to rethink the role of phenolics in plants, that they may be more affected by photodamage than by action of herbivores. The authors proposed that phenolics may be low because there is low risk to photodamage and therefore the 
metabolites are not required and not because of defense against herbivores. Although it was observed that there is a moderate to very strong inverse correlation between the FSIC and the concentration of total phenol and flavonoids in the leaves of the Lansium domesticum plants (Fig 4A and 4B), this does not mean that one causes the other.
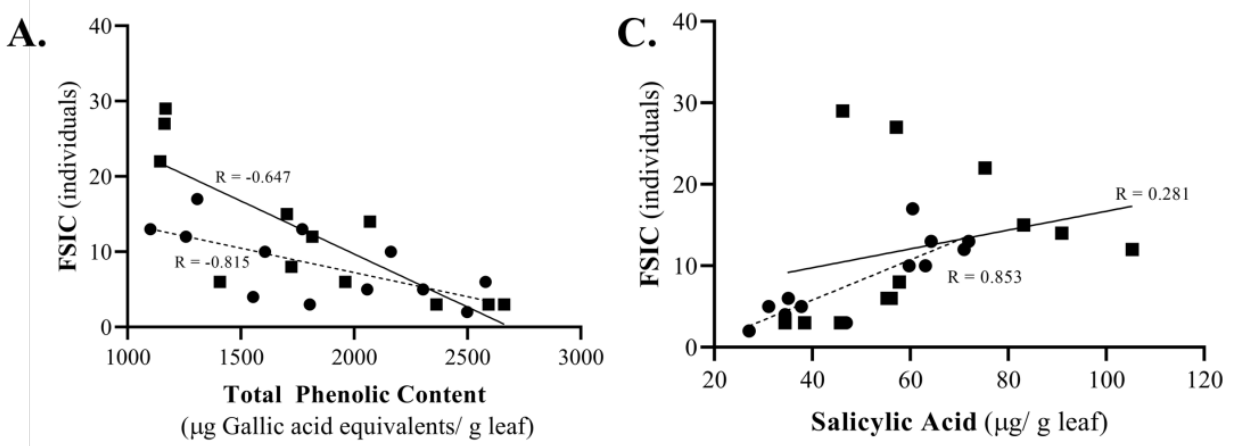

B.
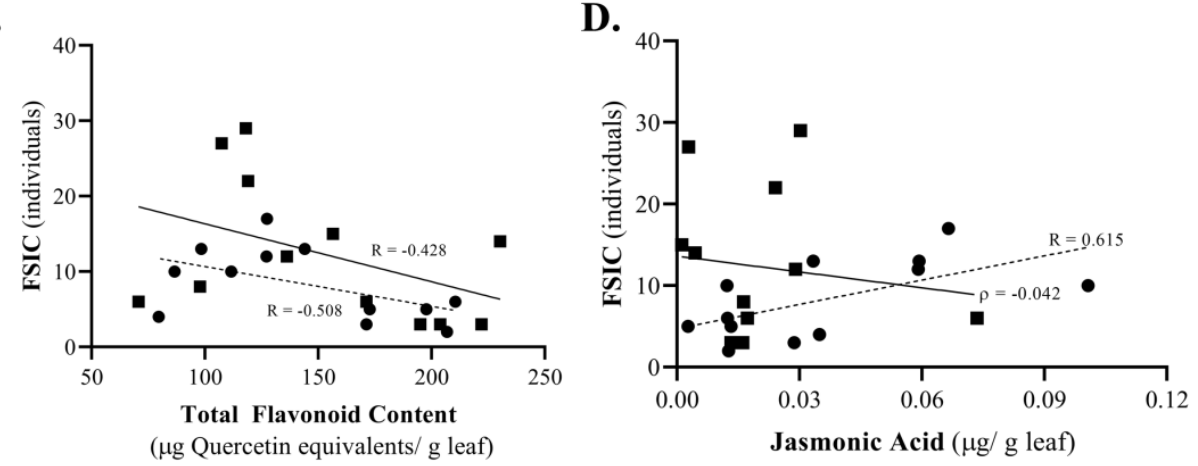

- with Trichoderma

- without Trichoderma

Figure 4. Comparisons of relationships between concentrations of total phenolic content (A), total flavonoid content (B), salicylic acid (C), and jasmonic acid (D) of the two treatments (with Trichoderma \& without Trichoderma) over the six-week observation period

It is possible that total phenolic concentrations decreased through time because the plants were maintained under partially shaded conditions inside an insect net tent and that its negative correlation with FSIC is just coincidental. If this is true, then it makes sense that all plants, regardless of treatment, would exhibit similar patterns of decrease in phenolics through time as they are continuously maintained under non-photodamaging conditions.

The overall decrease of the concentration of the phenolic and flavonoid groups may also be viewed as a natural optimization response of the plant to environmental variations even while there is continuous insect attack (Pieterse et al., 2012). That is besides the toxicity of high phenolic compounds concentration 
in the cell and the very resource-consuming whole range of adaptive responses that may be triggered due to the presence of the insect pests, often sacrificing even growth and development (Walters and Heil 2007).

Supporting the previously mentioned hypothesis that defense metabolites other than the members of the phenolic group is the example of the increased production of camalexin or 3-thiazol-2'-yl-indole that was shown to be induced by Trichoderma inoculation in Arabidopsis thaliana (Contreras-Cornejo et al., 2011). Camalexin is an example of indole derivatives that inhibit growth of pathogenic fungi like Botrytis cinerea and Cochliobolus carbonum, and the bacterium Pseudomonas syringae. Indole alkaloid camalexin has also been demonstrated to be involved in the resistance and defense mechanism of $A$. thaliana against Myzus persicae, a phloem-feeding green peach aphid (Kettle et al., 2013). Meanwhile, indole glucosinolates concentrations are also important in plants defense against plant herbivores.

For lanzones plants, the concentration of a specialized terpenoid found in lanzones trees called lansiolic acid that has been found to have a strong insect feeding deterrence activity (Mayanti et al., 2011; Ragasa, Labrador and Rideout, 2006; Arnason, Guillet, and Durst 2004) is a highly possible candidate for the secondary metabolite which could have had an increased production. ContrerasCornejo et al. (2018) further noted the positive relationship of Trichoderma inoculation and the observed increase in JA levels and volatile terpene emission.

Since there is a lack of available tools to dissect and quantify all of the possible secondary metabolites which can/may be produced by the lanzones plant, the relationship of the hormones and the pest can be looked into instead for possible explanations. Figures $4 \mathrm{C}$ and $4 \mathrm{D}$ show the correlations of the FSIC and the concentration of the two commonly implicated plant hormones during insect herbivory.

Plant detection of phloem-feeding insects, like Unaspis mabilis, is similar to their detection of pathogens, since these phloem-feeding insects create minimal plant tissue injury. The jasmonic acid-dependent and the salicylic aciddependent pathways regulate the plant's responses to this kind of attacker (Walling 2000). Figure 4D shows that there is a strong and significant relationship between JA and the FSIC $(\mathrm{R}=0.615, \mathrm{p}=0.0332)$ for the seedlings treated with Trichoderma. The same can be observed with the relationship of the same treatment with the concentration of SA $(R=0.8534, p=0.0004)$. On the other hand, the FSIC in seedlings that were not inoculated with Trichoderma showed a very weak and insignificant correlation with $\left.\mathrm{JA}_{(\mathrm{e}}=0.042, \mathrm{p}=0.897\right)$, and a weak correlation with SA $(\mathrm{R}=0.281, \mathrm{p}=0.376)$.

Since all the relationships between JA, SA, time, and FSIC are all weak and insignificant in the treatment without Trichoderma, while all the relationships between the same four parameters observed in the treatment inoculated with Trichoderma are strong, significant, and positive, it can be surmised that for lanzones plants infested with scale insects, the concentrations of both JA and SA are modulated by the inoculation of Trichoderma and that these 
were contributory to alleviating the infestation. These observations support the earlier hypothesis that both SA and JA play key roles in scale-insect defense response in the lanzones plant. This is consistent with the results of the study of Contreras-Cornejo et al. (2011) that both JA and SA accumulation was observed in A. thaliana inoculated with Trichoderma. In addition, multiple studies have shown that jasmonic acid and its derivatives can induce the production and accumulation of other secondary defense metabolites including saponin (Hu et al., 2003), terpenoid indole alkaloid (Goldhaber-Pasillas, Mustafa and Verpoorte, 2014), and terpenoid-based resins (Martin et al., 2002), that like the lansiolic acid, are noted for their role in plants' defenses.

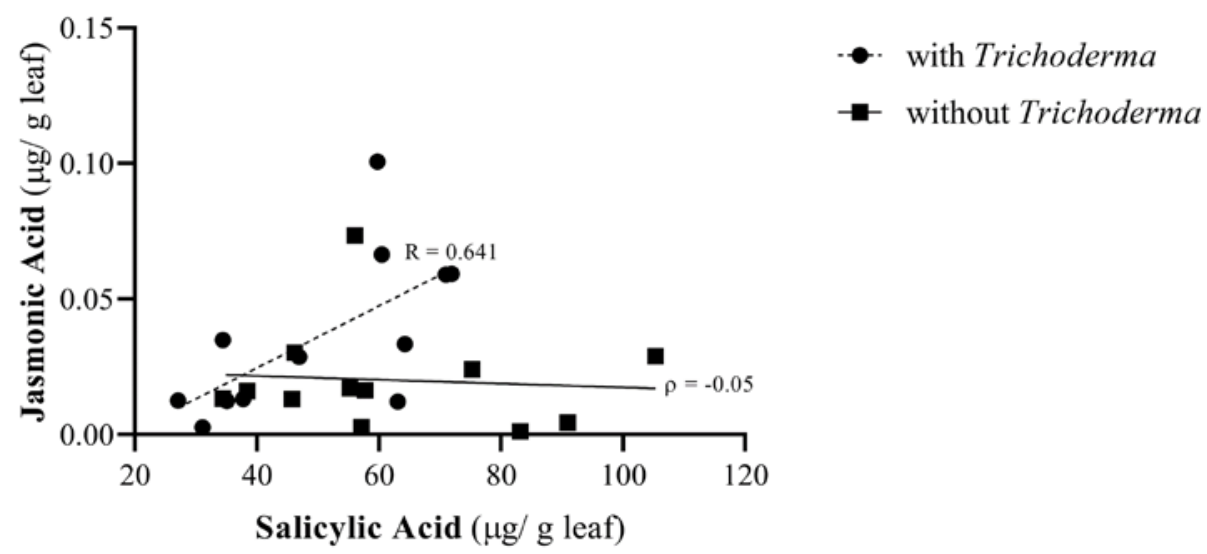

Figure 5. Correlation of salicylic acid and jasmonic acid concentrations in Lansium domesticum leaves infested with Unaspis mabilis in treatments with and without Trichoderma.

Figure 5 shows that there is a strong and significant correlation between $\mathrm{SA}$ and JA in plants inoculated with Trichoderma $(\mathrm{R}=0.641, \mathrm{p}=0.0247)$. Meanwhile, a very weak and insignificant association was observed between SA and JA in plants that were not inoculated with the same fungus ${ }_{e}=-0.056$, $\mathrm{p}=0.863$ ). These observations suggest a more refined tuning action of JA and SA in the presence of the Trichoderma inoculant. In these seedlings, the scale insect infestation declined through time and the seedlings were able to recover. This suggests that Trichoderma-inoculation was able to subvert the decoy defense of the insect by inducing the JA pathway thus allowing integration of both SA- and JA-mediated signaling pathways. This enabled the plant to launch effective defenses to antagonize insect performance.

While the Trichoderma-mediated subversion of the insect's "decoy defense" can effectively explain the observed concentrations of SA and JA in both the inoculated and uninoculated groups, possible SA-JA positive crosstalk may have still played an important role. This is because even with the "decoy 
defense hypothesis," SA-dependent defenses still contribute, although weakly, to the plant's overall defense strategy (Zhu-Salzman et al., 2004). The significant positive correlation between the JA and SA concentrations shown in the present study for Trichoderma-inoculated seedlings (Fig. 5) may still suggest that there is positive crosstalk between these two pathways and that they may have cooperated in order to launch effective defense responses against the pest. It is still possible that the cooperation of these two pathways lead to the production of a wide array of defense metabolites that all contribute to resistance against the lanzones scale insect.

According to Martinez-Medina et al. (2013), reports of the necessity for JA in Trichoderma-induced systemic resistance are consistent in systems using Arabidopsis thaliana as model. Several other studies have been conducted that implicate the JA signaling pathway in the Trichoderma-mediated induced systemic resistance (TISR) in plants. In the study by Shoresh, Yedidia and Chet (2005), the JA signaling pathway was identified as the signal transduction pathway in the induction of systemic resistance by Trichoderma asperellum T203 in cucumber. The study by Segarra et al. (2009) likewise confirms the role of the JA pathway in Trichoderma-mediated ISR. In addition, Martinez-Medina et al. (2013) also reported that in tomato models, SA- regulated pathways are also required for a successful TISR development. Other studies show the capability of Trichoderma to induce resistance by triggering both JA- and SA-mediated defense response, and that the SA and/or JA/ET pathways may overlap depending on several conditions (Saldajeno et al., 2014; Yoshioka et al., 2012; Tucci et al., 2011; Segarra et al., 2007). Gallou, Cranenbrouck and Declerck (2009) also reported that the potato plants inoculated with Trichoderma harzianum when challenged with Rhizoctonia solani displayed a defense response that was dependent on both JA/ET and SA pathways.

Altogether, the results of the present study demonstrate that Trichoderma inoculation induced the JA pathway and played an important role in defense against lanzones scale insect. Furthermore, the interaction of the JA pathway with the SA-dependent pathway is fine-tuned in the presence of Trichoderma.

\section{CONCLUSIONS}

The capacity of Trichoderma spp. to be a protective agent has been clearly demonstrated. The healthy plants inoculated with the fungus have shown better resistance when challenged by Unaspis mabilis infestation. This was determined through the observation of morphological parameters of the plants tested to assess their general health condition, as well as the population changes of the scale insects during a four-month observation period. In general, the plants inoculated with Trichoderma have displayed higher leaf number, and lower insect population than that of the seedlings that were not inoculated with the myco-biocontrol agent.

The capacity of Trichoderma spp. to alleviate scale insect infestation, on the other hand, was tested through the simultaneous observation of the same 
morphological parameters, and phytochemical parameters. It was observed that the plants inoculated with Trichoderma have shown better performance against the infestation of the $U$. mabilis since the population of scale insects was significantly lower and was growing more slowly.

The two groups of plant defense metabolites (phenols and flavonoids) concentration were observed to be decreasing over time. It was proposed that this could be due to other environmental cues (non-photodamaging conditions) and not just a response to insect attack. It is also probable that other compounds were involved or that it is not the total concentration of the groups that is important for an optimized defense but the characteristic and the blend of the specific compounds.

In addition, the phytohormones JA and SA, have been observed to be affected by the presence of the Trichoderma inoculation. Drawing from previous studies, three hypotheses were proposed to explain the effect of the Trichoderma inoculation on these two plant hormones in the case of Trichoderma - $L$. domesticum - U. mabilis interaction:

1.) the scale insects have the capacity to increase the production of SA inside the plants, thereby blocking the JA-dependent defense pathways, while the inoculation of Trichoderma to the plants causes an increased JA production to counter this SA increase;

2.) the inoculation of Trichoderma may have had a simultaneous effect on the concentration of SA and JA in order to utilize both the SAdependent defense response and the JA-dependent defense pathways of the plant; or

3.) a combination of both.

\section{ACKNOWLEDGEMENTS}

The authors would like to thank the Institute of Biological Sciences and the Institute of Chemistry of the University of the Philippines Los Baños for supporting this project. Likewise, thanks are given to Dr. Virginia Cuevas for the Trichoderma inoculant used in the study, and to Ms. Rachelyn Ann Araña, for the advice given on statistical analyses. Gratitude is also due to Mr. Mark Lorenz P. Mangosing, without whom this would not be possible.

\section{REFERENCES}

Arnason JT, Guillet G, \& Durst T. (2004). Phytochemical diversity of insect defenses in tropical and temperate plant families. In Carde RT, Millar JC, editors. Advances in Insect Chemical Ecology (pp. 1-20). Cambridge University Press.

Brion, AC. (2016). Fighting lanzones' scale insect with Chilocorus nigrita. DA-BAR Digest 18(2). Retrieved from: https://www.bar.gov.ph/index.php/digesthome/digest-archives/771-2016-2nd-quarter/5827-fighting-lanzones-scale-insectwith-chilocorus-nigrita

Close DC \& McArthur C. (2002). Rethinking the role of many plant phenolics protection from photodamage not herbivores? Oikos 99: 166-172. 
Cock IE. (2011). An examination of the medicinal potential of Scaevola spinescens: Toxicity, antibacterial and antiviral activities. Pharmacogn. Res, 3(2), 85-94.

Conrath U, Beckers GJM, Flors V, Garcia-Agustin P, Jakab G, Mauch F, Newman MA, Pieterse CMJ, Benoit P, Pozo MJ, Pugin A, Schaffrath U, Ton J, Wedehenne D, Zimmerli, \& Mauch-Mani B. (2006). Priming: getting ready for battle. Mol Plant Microbe Interact, 19(10), 1062-1071.

Contreras-Cornejo HA, Marcias-Rodriguez L, Beltran-Pena E, Herrera-Estrella A, \& Lopez-Bucio J. (2011). Trichoderma-induced plant immunity likely involves both hormonal- and camalexin-dependent mechanisms in Arabidopsis thaliana and confers resistance against necrotrophic fungus Botrytis cinerea. Plant Signal Behav, 6(10), 1554-1563.

Contreras-Cornejo, H. A., Macías-Rodríguez, L., del-Val, E., \& Larsen, J. (2018). The root endophytic fungus Trichoderma atroviride induces foliar herbivory resistance in maize plants. Applied Soil Ecology, 124(October), 45-53.

Dhandhukia PC \& Thakkar VR. (2008). Separation and quantitation of jasmonic acid using HPTLC. J Chromatogr Sci, 46, 320-324

Evans WC. (1989). Trease and Evans Pharmacognosy. 13th Ed. Bailliere Tindall, London.

Frost CJ, Mescher ME, Carlson JE, \& De Moraes CM. (2008). Plant defense priming against herbivores: Getting ready for a different battle. Plant Physiol, 146, 818824.

Gallou A, Cranenbrouck S, \& Declerck S. (2009). Trichoderma harzianum elicits defence response genes in roots of potato plantlets challenged by Rhizoctonia solani. Eur J Plant Pathol, 124, 219-230.

Glazebrook J. (2005). Contrasting mechanisms of defense against biotrophic and necrotrophic pathogens. Annu Rev Phytopathol, 43, 205-227.

GMA News Online. (2008, October 23). Chemicals no match for lanzones pest. Retrieved from http://www.gmanetwork.com/news

Goldhaber-Pasillas GD, Mustafa NR, \& Verpoorte R. (2014). Jasmonic acid effect on the fatty acid and indole terpenoid alkaloid accumulation in cell suspension cultures of Catharantus roseus. Molecules, 9, 10242-10260.

Harborne JB, Baxter H, \& Moss GP. (1999). Phytochemical dictionary: A handbook of bioactive compounds from plants (pp. 359). $2^{\text {nd }}$ Ed. Taylor \& Francis.

Harman GE. (2000). Myths and dogmas of biocontrol: changes in perceptions derived from research on Trichoderma harzianum T-22. Plant Dis. 84, 377-393.

Harman GE. (2001). Microbial tools to improve crop performance and profitability and to control plant diseases. In D. D.-S. Tzeng \& J.W. Huang (eds.), In Proceedings of International Symposium on Biological Control of Plant Diseases for New Century - Mode of Action and Application Technology (pp. 71-84). National Chung Hsing University, Taichung City, Taiwan.

Harman GE. (2006). Overview of mechanisms and uses of Trichoderma spp. Phytopathology, 96(2), 190-194.

Harman GE, Bjorkman T, Ondik K, \& Shoresh M. (2008) Changing paradigms on the mode of action and uses of Trichoderma spp. for biocontrol. Outlooks on Pest Management, 19(1), 24-29.

Harman GE, Howell CR, Viterbo A, Chet I, \& Lorito M. (2004). Trichoderma species - opportunistic, avirulent plant symbionts. Nat Rev Microbiol, 2, 43-56.

Harman GE, Shoresh M. (2007). The mechanism and applications of opportunistic plant symbionts. In M. Vurro \& J. Gressels (eds.), In Novel Biotechnologies for 
Biocontrol Agent Enhancement and Management (pp. 131-153). Springer, Amsterdam, The Netherlands.

Hu X, Neil S, Cai W, \& Tang Z. (2003). Hydrogen peroxide and jasmonic acid mediate oligogalacturonic acid-induced saponin accumulation in the suspension-cultured cells of Panax ginseng. Physiol Plant, 118, 414-421.

Kaloshian I, \& Walling LL. (2005). Hemipterans as plant pathogens. Annu Rev Phytopathol, 43, 491-521

Kempema LA, Cui X, Holzer FM, \& Walling LL. (2007). Arabidopsis transcriptome changes in response to phloem-feeding silverleaf whitefly nymphs: similarities and distinctions in responses to aphids. Plant Physiol, 143, 849-865.

Kettle GJ, Drurey C, Schoonbeek H, Maule A, \& Hogenhout SA. (2013). Resistance of Arabidopsis thaliana to the green peach aphid, Myzus persicae, involves camalexin and is regulated by microRNAs. New Phytol, 198(4), 1178-1190.

Kiranmai M, Kumar CBM, \& Ibrahim M. (2011). Comparison of total flavonoid content of Azadrirachta indica root bark extract prepared by different extraction method. Res J Pharm Biol Chem Sci, 2(3), 254-261

Lit IL, \& Barbecho N. (2014). A new species of armored scale insect (Hemiptera: Diaspididae) on Lansium domesticum trees in the Philippines. ARTHROPODA Generalis, 4, 1-7.

Lo CT, \& Lin CY. (2002). Screening strains of Trichoderma spp for plant growth enhancement in Taiwan. Plant Pathology Bulletin, 11, 215-220.

Martin D, Tholl D, Gershenzon J, \& Bohlmann J. (2002). Methyl jasmonate induces traumatic resin ducts, terpenoid resin biosynthesis, and terpenoid accumulation in developing xylem of Norway spruce stems. Plant Physiol, 12, 1003-1018

Martinez-Medina A, Fernandez I, Sanchez-Guzman MJ, Jung SC, Pascual JA, \& Pozo MJ. (2013). Deciphering the hormonal signaling network behind the systemic resistance induced by Trichoderma harzianum in tomato. Front Plant Sci, 4(206), $1-12$.

Mayanti T, Natawigena WD, Supratman U, \& Tjokronegoro R. (2011). Antifeedant terpenoid from the seeds and bark of Lansium domesticum Corr cv. Kokossa (Meliaceae). Molecules, 16, 2785-2795.

Moran PJ, \& Thompson GA. (2001). Molecular responses to aphid feeding in Arabidopsis in relation to plant defense pathways. Plant Physiol, 125, 10741085.

Pieterse CMJ, Van Der Does D, Zamioudis C, Leon-Reyes A, \& Vanwees CM. (2012). Hormonal modulation of plant immunity. Annu Rev Cell Dev Biol, 28, 489-521.

Pieterse CMJ, Van Pelt JA, Ton J, Parchmann S, Mueller MJ, Buchala AJ, Métraux JP, \& Van Loon LC. (2000) Rhizobacteria-mediated induced systemic resistance (ISR) in Arabidopsis requires sensitivity to jasmonate and ethylene but is not accompanied by an increase in their production. Physiol Mol Plant Pathol, 57, $123-134$.

Provido, N.T. (2007) How to control lanzones pest. Philippine Headline News Online; MANILA (STAR), 26 September 2007. Available from: http://www.newsflash.org/2004/02/si/si002421.htm (accessed 02 May 2019)

Ragasa CY, Labrador P, \& Rideout JA. (2006). Antimicrobial terpenoids from Lansium domesticum. Philipp Agric Sci, 8(1), 101-105.

Rodriguez-Saona C, \& Thaler JS. (2005). The jasmonate pathway alters herbivore feeding behavior: consequences for plant defences. Entomol Exp Appl, 115(1), 125-134. 
Rojo E, Solano R \& Sanchez-Serrano JJ. (2003). Interactions between signal- ing compounds involved in plant defense. J Plant Growth Regul 22, 82-98

Saldajeno MGB, Naznin HA, Elsharkawy MM, Shimizu M \& Hyakumachi M. (2014). Enhanced resistance of plants to disease using Trichoderma. In V. Gupta, M. Schmoll, A. Estrella, R. Upadhyay, I. Druzhinina, \& M. Tuohy, ed. Biotechnology and Biology of Trichoderma (pp. 474-493). Waltham, MA: Elsevier.

Segarra G, Casanova E, Bellido D, Odena MA, Oliveira E, \& Trillas I. (2007). Proteome, salicylic acid, and jasmonic acid changes in cucumber plants inoculated with Trichoderma asperellum strain T34. Proteomics, 7, 3943-3952.

Segarra G, Van Der Ent S, Trillas I, \& Pieterse CMJ. (2009). MYB72, a node of convergence in induced systemic resistance triggered by a fungal and a bacterial beneficial microbe. Plant Biol, 11, 90-96.

Shoresh M, Yedidia I, \& Chet I. (2005). Involvement of jasmonic acid/ethylene signaling pathway in the systemic resistance induced in cucumber by Trichoderma asperellum T203. Phytopathology, 95, 76-84

Singleton VL, Orthofer R, \& Lamuela-Raventos RM. (1999). Analysis of total phenols and other oxidation substrates and antioxidants by means of Folin-Ciocalteu reagent. Methods Enzymol, 299, 152-178.

Smith JL, De Moraes CM, \& Mescher MC. (2008). Jasmonate- and salicylic-mediated plant defense responses to insect herbivores, pathogens and parasitic plants. Pest Manag Sci, 65, 497-503

Thaler JS, Agrawal AA, \& Halitschke R. (2010). Salicylate-mediated interactions between pathogens and herbivores. Ecology, 91(4), 1075-1082.

Thompson GA, \& Goggin FL. (2006). Transcriptomics and functional genomics of plant defence induction by phloem-feeding insect. J. Exp. Bot., 57(4), 755-766.

Tucci M, Ruocco M, De Masi L, De Palma M, \& Lorito M. (2011). The beneficial effect of Trichoderma spp. on tomato is modulated by the plant genotype. Mol. Plant Pathol, 12, 341-354.

Ueda J, \& Miyamoto K. (1994). Separation of a new type of plant growth regulator, jasmonates, by chromatographic procedures. J Chromatogr A, 658, 129-142.

Walling LL. (2000). The myriad plant responses to herbivores. J Plant Growth Regul, 19: 195-216.

Walling LL. (2008). Avoiding effective defenses: Strategies employed by phloemfeeding insects. Plant Physiol, 146: 859-866.

Walters D, \& Heil M. (2007). Costs and trade-offs associated with induced resistance. Physiol Mol Plant Pathol, 71, 3-17.

War A, Paulraj M, Ahmad T, Buhroo A, Hussain B, Ignacimuthu S, \& Sharma H. (2012). Mechanisms of plant defense against insect herbivores. Plant Signal Behav, 7(10), 1306-1320.

Warrier RR, Paul M, \& Vineetha MV. (2013). Estimation of salicylic acid in eucalyptus leaves using spectrophotometric methods. Genetics and Plant Physiology, 3, 1-2.

Watson, G. W. (2015). Synonymy in the armoured scale insect genus Unaspis MacGillivray (Hemiptera: Coccomorpha: Diaspididae). Zootaxa, 3980(3), 450.

Xiao L, Carrillo J, Siemann E, \& Ding J. (2019). Herbivore-specific induction of indirect and direct defensive responses in leaves and roots. AoB PLANTS 11: plz003; doi: 10.1093/aobpla/plz003. 
Yedidia I, Srivastva AK, Kapulnik Y \& Chet I. (2001). Effect of Trichoderma harzianum on microelement concentrations and increased growth of cucumber plant. Plant Soil, 235, 235-242

Yoshioka Y, Ichikawa H, Naznin HA, Kogure A, \& Hyakumachi M. (2012). Systemic resistance induced in Arabidopsis thaliana by Trichoderma asperellum SKT-1, a microbial pesticide of seedborne diseases of rice. Pest Manage Sci, 68, 60-66.

Zarate JT, Caasi-Lit MT, Cuevas VC \& Cocjin GT. (2015). Terrestrial arthropod profile and soil microbial population dynamics on cabbage cropping as affected by application of Trichoderma microbial inoculant (TMI) in Quezon, Sariaya, Philippines. Journal of Environmental Science and Management, 18(2), 10-22.

Zarate SI, Kempema LA \& Walling LL. (2007). Silverleaf whitefly induces salicylic acid responses and represses effectual jasmonic responses in Arabidopsis. Plant Physiol, 143, 866-875.

Zhou, D., Huang, X. F., Guo, J., dos-Santos, M. L., \& Vivanco, J. M. (2018). Trichoderma gamsii affected herbivore feeding behaviour on Arabidopsis thaliana by modifying the leaf metabolome and phytohormones. Microbial Biotechnology, 11(6), 1195-1206.

Zhu-Salzman K, Salzman RA, Ahn JE, \& Koia H. (2004). Transcriptional regulation of sorghum defense determinants against a phloem-feeding aphid. Plant Physiol, 134(1), 420-431. 